Supporting Information for:

\title{
Protein Cross-Linking Capillary Electrophoresis for Protein-Protein Interaction Analysis
}

Claire M. Ouimet ${ }^{\dagger}$, Hao Shao ${ }^{\S}$, Jennifer N. Rauch ${ }^{\S}$, Mohamed Dawod ${ }^{\dagger}$, Bryce Nordhues ${ }^{\|}$, Chad A. Dickey"l, Jason E. Gestwicki ${ }^{\S}$, Robert T. Kennedy ${ }^{\dagger, \ddagger} *$

${ }^{\S}$ Department of Pharmaceutical Chemistry and the Institute for Neurodegenerative Disease, 675 Nelson Rising Ln, University of California at San Francisco, San Francisco, California 94158, United States

${ }^{\|}$Department of Molecular Medicine, University of South Florida, 4001 E. Fletcher Ave., MDC 36, Tampa, Florida 33613, United States

${ }^{\dagger}$ Department of Chemistry, University of Michigan

930 N. University Ave, Ann Arbor, Michigan, 48109, United States

${ }^{\ddagger}$ Department of Pharmacology, University of Michigan

1150 W. Medical Center Dr., Ann Arbor, Michigan, 48109, United States

*Corresponding author

E-mail: rtkenn@umich.edu

Tel: $734-615-4363$

Fax: 745-615-6462 


\section{Supporting Information:}

Figure S-1. Free solution electrophoresis of chaperone complexes Hsp70-488-Bag3 and Hsp90488 homodimer.

Table S-1. Quantitation of resolution of free proteins and protein complexes.

Figure S-2. Capillary gel electrophoresis of lysozyme immunocomplex.

Table S-2. Dependence of Hsp70-488-Bag3 complex detected on concentration of formaldehyde and cross-linking reaction time.

Table S-3. Dependence of Hsp90-488 complex detected on concentration of formaldehyde and cross-linking reaction time.

Table S-4. Dependence of FITC-lysozyme-antibody complex detected on concentration of formaldehyde and cross-linking reaction time.

Figure S-3. Saturation binding assays for Hsp70-488-Bag3 at different 1\% formaldehyde reaction times.

Table S-5. Dependence of measured $\mathrm{K}_{\mathrm{d}}$ of Hsp70-488-Bag3 interaction on cross-linking reaction time using $1 \%$ formaldehyde.

Figure S-4. Calorimetric isothermal-titration measurement of FITC-lysozyme interaction with anti-lysozyme.

Figure S-5. FCPIA data of small molecule Hsp70-488-Bag3 inhibitors JG-98, JG-231 and JG311. 
Figure S-1. Free solution electrophoresis of chaperone complexes Hsp70-488-Bag3 (A) and Hsp90-488 homodimer (B). The electrophoresis buffer was $10 \mathrm{mM}, \mathrm{pH} 10$ borate.

A

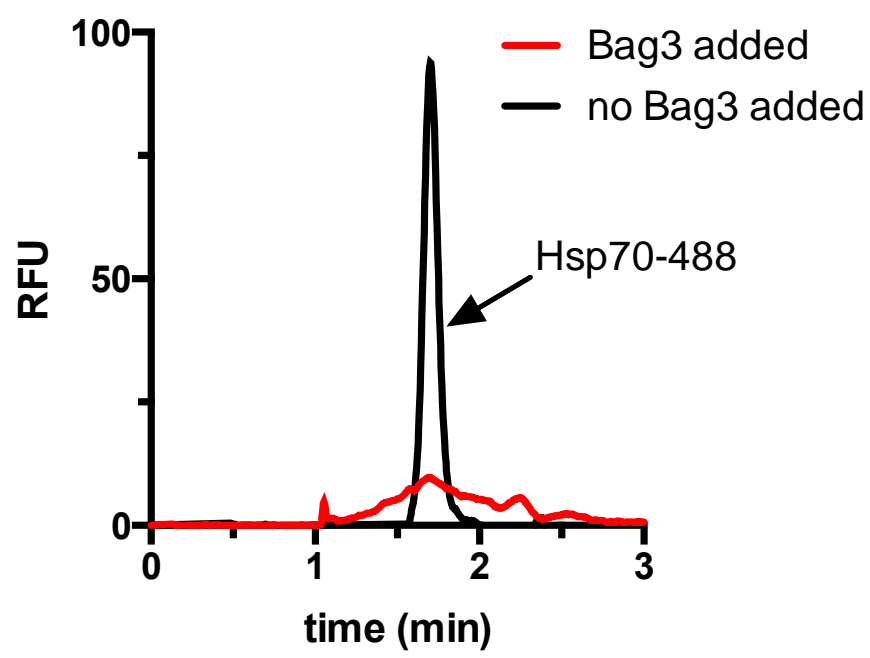

B

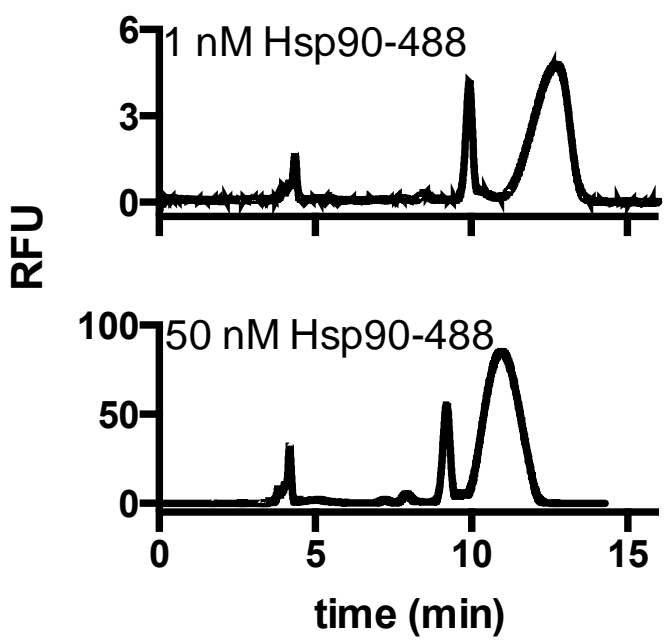


Table S-1. Resolution of free proteins from protein complexes.

\begin{tabular}{|l|l|}
\hline Complex & Resolution \\
\hline Hsp70-Bag3 & 1.0 \\
\hline Hsp90 homodimer & 2.0 \\
\hline Lysozyme-anti-lysozyme & 1.1 \\
\hline
\end{tabular}


Figure S-2. Capillary gel electrophoresis separation of FITC-lysozyme immunocomplex.

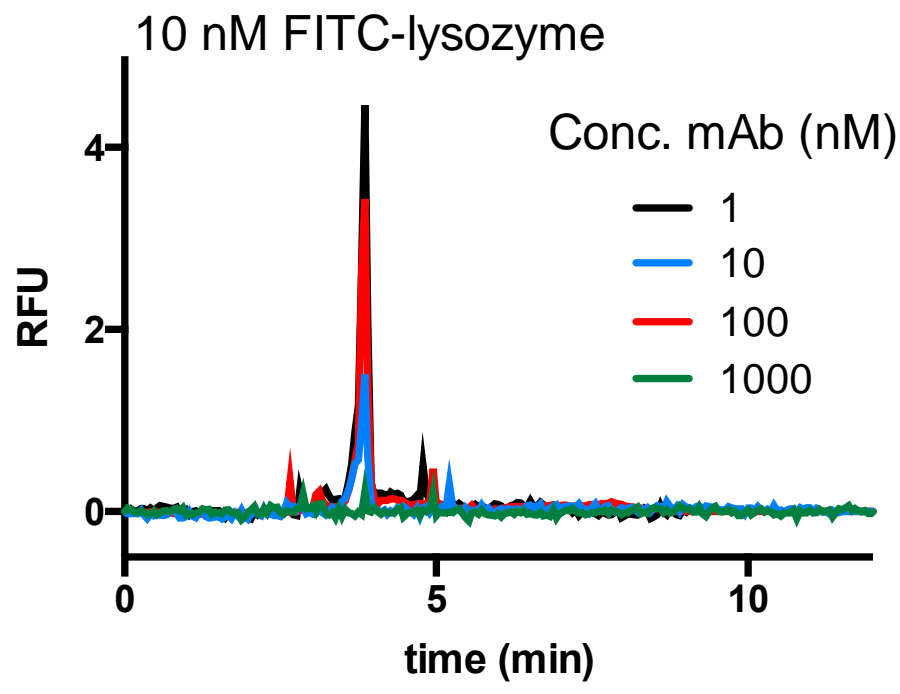


Table S-2. Dependence of $25 \mathrm{nM}$ Hsp70-488 and $100 \mathrm{nM}$ Bag3 on Hsp70-Bag3 complex detected on concentration of formaldehyde and cross-linking reaction time. Error is range of two trials.

\begin{tabular}{|c|r|r|r|r|r|}
\hline \multicolumn{7}{|c|}{ Percent complex (\%) } \\
\hline $\begin{array}{c}\text { formaldehyde } \\
\text { concentration } \\
(\% \text { w/v) }\end{array}$ & \multicolumn{6}{|c|}{ Reaction time (min) } \\
\cline { 2 - 6 } & $\mathbf{0 . 5}$ & $\mathbf{5}$ & $\mathbf{1 0}$ & $\mathbf{3 0}$ & \multicolumn{1}{c|}{$\mathbf{0}$} \\
\hline $\mathbf{0 . 0 5 0}$ & $20 \pm 3$ & $21 \pm 8$ & $30 \pm 17$ & $38 \pm 3$ & $29 \pm 6$ \\
\hline $\mathbf{0 . 5 0}$ & $19 \pm 5$ & $40 \pm 13$ & $60 \pm 9$ & $74 \pm 9$ & $46 \pm 13$ \\
\hline $\mathbf{1 . 0}$ & $22 \pm 9$ & $46 \pm 24$ & $73 \pm 10$ & $73 \pm 6$ & $24 \pm 3$ \\
\hline $\mathbf{2 . 5}$ & $31 \pm 2$ & $62 \pm 17$ & $73 \pm 2$ & $75 \pm 8$ & $44 \pm 5$ \\
\hline
\end{tabular}

Table S-3. Dependence of $50 \mathrm{nM}$ Hsp90-488 on complex detected on concentration of formaldehyde and cross-linking reaction time. Error is range of two trials.

\begin{tabular}{|c|r|r|r|r|}
\hline \multicolumn{5}{|c|}{ Percent complex (\%) } \\
\hline $\begin{array}{c}\text { formaldehyde } \\
\text { concentration } \\
(\boldsymbol{\%} \text { w/v) }\end{array}$ & \multicolumn{4}{|c|}{ Reaction time (min) } \\
\cline { 2 - 5 } & $\mathbf{0 . 5}$ & $\mathbf{5}$ & \multicolumn{1}{c|}{$\mathbf{1 0}$} & \multicolumn{1}{c|}{$\mathbf{5 0}$} \\
\hline $\mathbf{0 . 0 5 0}$ & $80 \pm 5$ & $77 \pm 1$ & $81.2 \pm 0.4$ & $71.4 \pm 0.9$ \\
\hline $\mathbf{0 . 5 0}$ & $77 \pm 5$ & $74 \pm 6$ & $73.3 \pm 0.6$ & $74.6 \pm 0.8$ \\
\hline $\mathbf{1 . 0}$ & $73 \pm 2$ & $79 \pm 10$ & $72 \pm 3$ & $73 \pm 6.5$ \\
\hline $\mathbf{2 . 5}$ & $78 \pm 6$ & $73 \pm 7$ & $64 \pm 4$ & $58 \pm 4$ \\
\hline
\end{tabular}

Table S-4. Dependence of $10 \mathrm{nM}$ FITC-lysozyme and $20 \mathrm{nM}$ antibody on complex detected on concentration of formaldehyde and cross-linking reaction time. Error is range of two trials.

\begin{tabular}{|c|r|r|r|r|}
\hline \multicolumn{5}{|c|}{ Percent complex (\%) } \\
\hline $\begin{array}{c}\text { formaldehyde } \\
\text { concentration } \\
(\% \mathbf{w} / \mathbf{)})\end{array}$ & $\mathbf{0 . 5}$ & $\mathbf{5}$ & $\mathbf{1 0}$ & \multicolumn{1}{c|}{$\mathbf{6 0}$} \\
\cline { 2 - 5 } & $27 \pm 15$ & $39 \pm 1$ & $34 \pm 7$ & $24 \pm 3$ \\
\hline $\mathbf{0 . 0 5 0}$ & $33 \pm 15$ & $37 \pm 8$ & $37 \pm 12$ & $39 \pm 11$ \\
\hline $\mathbf{0 . 5 0}$ & $39 \pm 15$ & $35 \pm 1$ & $40 \pm 9$ & $34 \pm 11$ \\
\hline $\mathbf{1 . 0}$ & $42 \pm 24$ & $27 \pm 8$ & $28 \pm 12$ & $23 \pm 4$ \\
\hline $\mathbf{2 . 5}$ & & &
\end{tabular}


Figure S-3. Saturation binding assays for Hsp70-488-Bag3 at different 1\% formaldehyde reaction times. All samples contain $25 \mathrm{nM}$ Hsp70-488, error bars are standard deviation $(\mathrm{n}=3)$.

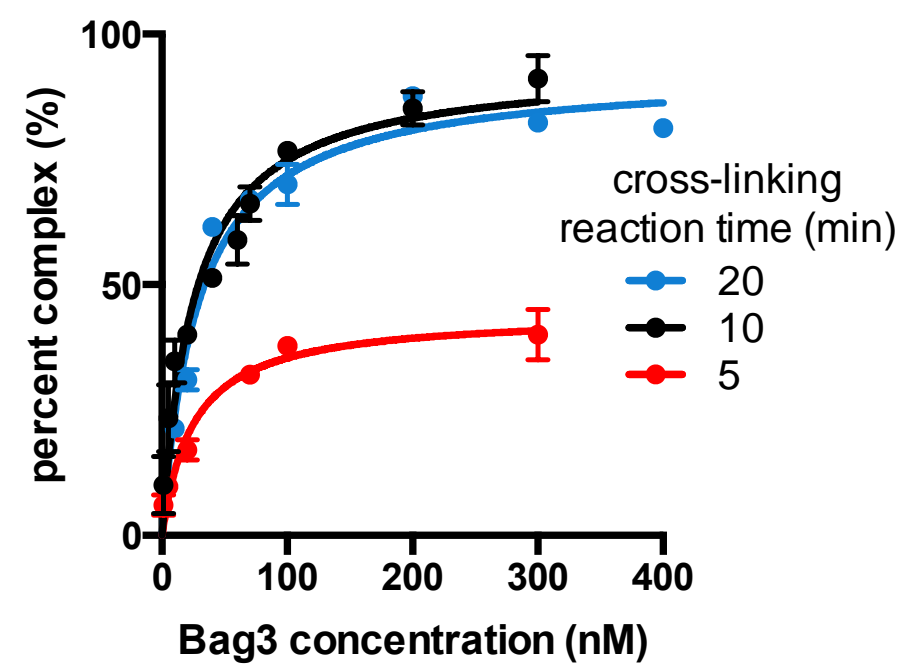

Table S-5. Dependence of measured $K_{d}$ of Hsp70-488-Bag3 interaction on cross-linking reaction time using $1 \%$ formaldehyde.

\begin{tabular}{|c|c|}
\hline Cross-linking reaction time (min) & $\mathbf{K}_{\mathbf{d}, \mathbf{H s p 7 0}-\mathrm{Bag} 3} \mathbf{( n M )}$ \\
\hline 5 & $25 \pm 8$ \\
\hline 10 & $25 \pm 5$ \\
\hline 20 & $29 \pm 6$ \\
\hline
\end{tabular}


Figure S-4. Calorimetric isothermal-titration measurement of FITC-lysozyme interaction with anti-lysozyme.

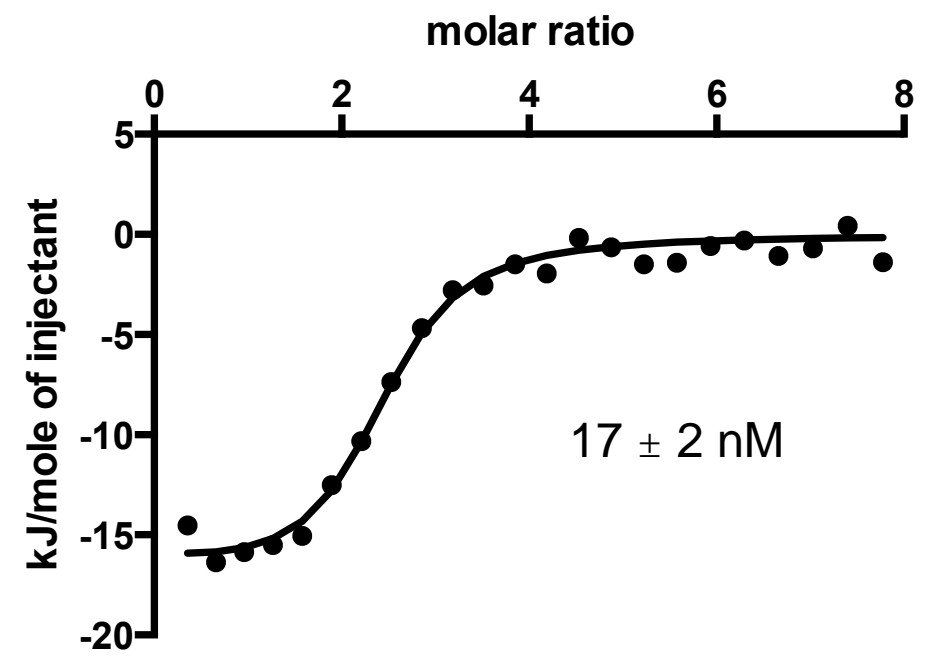


Figure S-5. FCPIA data of small molecule Hsp70-Bag3 inhibitors JG-98, JG-231 and JG-311. Error bars are range of two trials.

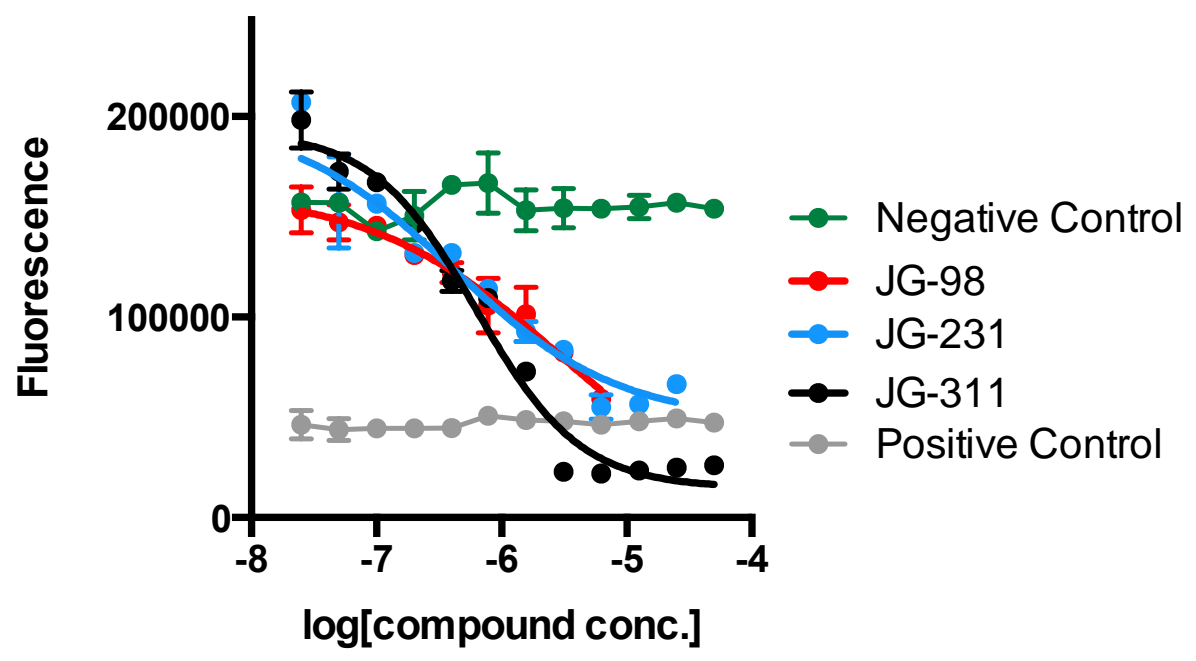

